\title{
Gestión Directiva Universitaria: Un Instrumento para su Evaluación
}

\section{University Management: An Assessment Instrument}

\author{
Carmen Bonnefoy ${ }^{1}$, Gamal Cerda, Shila Peine, \\ María Durán, Yeleni Ponce ${ }^{2}$
}

\begin{abstract}
Resumen
La investigación da cuenta del proceso de construcción y validación de un instrumento para medir gestión de directivos universitarios desde un enfoque basado en competencias, por ello se exhiben las propiedades psicométricas y la dimensionalidad de la Escala de Evaluación de la Gestión Directiva (E.E.G.D.) en cuanto a su proceso de diseño, elaboración y aplicación. Se presenta un análisis para la validez de contenido, a través de la técnica de jueces expertos y un análisis factorial para examinar su validez de constructo, considerando los índices de confiabilidad de la misma. Los resultados demuestran que la escala posee adecuadas características psicométricas, pertinentes al constructo examinado, constituyéndose en un instrumento relevante para los procesos de evaluación y acreditación universitaria.
\end{abstract}

Palabras Claves: Gestión Directiva, Evaluación de Calidad, Competencias

\begin{abstract}
This research presents the building and the validity process of an instrument designed to measure university authorities' management from an approach based on skills. Psychometric properties and the dimensionality of the Executive Management Evaluation Scale (EMES) related to its design process, elaboration and application are presented.

By means of the expert judge technique, an analysis of the validity of contents is indicated, as well as a factorial analysis in order to assess the validity of the construct, considering a reliability index.

Results show that the scale has accurate psychometric features, which are relevant to the examined construct, turning this instrument into a useful tool for the processes of assessment and university accreditation.
\end{abstract}

Key Words: Executive management, quality assessment, skills

1 Psicóloga, Doctorado en Psicología, Universidad de Chile. Decana Fac. de Psicología Universidad San Sebastián. email: cbonnefoy@uss.cl

2 Alumnos tesistas de la Carrera de Psicología de la Universidad San Sebastián 


\section{Introducción}

Los cambios que trae aparejada la llamada sociedad de la información, la creciente demanda de mayor calidad y de actualización o formación continua, el empleo de nuevos métodos de formación, la necesidad de aunar la especialización con el cultivo de las ciencias multidisciplinarias, la autonomía universitaria, son desafíos a los cuales las instituciones de educación superior no pueden permanecer ajenas y, no sólo han de responder a ellas como ha sido hasta ahora, manteniendo y aumentando en forma perpetua la brecha y el rezago, sino más bien deben ser capaces de vislumbrar nuevos horizontes de desarrollo, nichos de formación emergentes, modalidades de educación a distancia o en el trabajo, obtención de recursos económicos y materiales cada vez más escasos, etc., que configuren un enfoque proactivo más que reactivo frente a las demandas del entorno: "Los nuevos valores sociales y los planteamientos competitivos que vive el contexto de la educación superior (incremento del número de titulaciones, recortes presupuestarios, florecimiento de centros privados y a distancia, etc.), así como la preocupación interna por alcanzar la excelencia académica, son algunas claves para entender la necesidad sentida de modificar los estilos de organización de la gestión universitaria" (Rebolloso, 2001, p. 13).

Es en este contexto donde las instituciones de educación superior tienen la oportunidad de demostrar no sólo su capacidad de adaptación, sino más bien su liderazgo social e intelectual. Para ello, se deben adoptar cambios drásticos tanto en la forma, como en el diseño de su organización interna y en las relaciones de ésta con la sociedad.

Ante tales demandas, las universidades tienen que tornar a instituciones que aprenden y que tienen el sello de la calidad total, que les permita afrontar y vislumbrar estos desafíos sin un deterioro de la enseñanza ni de la formación profesional e investigadora, que hasta ahora ha sido su sello distintivo. La autonomía y la independencia son esenciales a la hora de estimular la buena docencia, lo mismo que los procesos de formación e investigación de las universidades. Esta autonomía va unida a la autogestión y autofinanciamiento. Para esto es necesario la implantación de planes de evaluación del rendimiento y de resultados, considerando factores tales como la eficiencia y el rendimiento social. Este proceso de evaluación deberá ser dinámico y su fin será la mejora de la institución en su globalidad.

Las universidades, como todas las instituciones, tienen una misión que cumplir, es decir, un conjunto de objetivos estratégicos que orientan su desarrollo en el tiempo y que indica el sentido de dirección y de logro. Es responsabilidad de sus directivos tomar las decisiones adecuadas para generar y administrar los recursos necesarios a fin de alcanzar los objetivos universitarios. Por esta razón, los directivos universitarios necesitan conocer cuán bien están desarrollando sus labores ejecutivas con el fin de corregir los aspectos que se requiere perfeccionar para realizar un desempeño eficaz y eficiente. (Valenzuela, 1999). La evaluación de las instituciones universitarias se ha limitado tradicionalmente a las personas: conocer el grado de satisfacción del alumnado, como usuario, a través de encuestas sobre el profesorado y sus materias, o evaluar su actividad investigadora a partir de la producción científica. Sin embargo, cada vez más, se demanda una evaluación globalizadora y procesal que permita determinar de qué forma la institución se aproxima a las metas preestablecidas y al desarrollo de la producción y distribución del conocimiento, lo que requiere evaluar la investigación, gestión y docencia. (Pérez, 2000).

En los últimos años, la evaluación de la calidad en las instituciones universitarias se ha convertido en un tema prioritario. Esta predisposición hacia la evaluación de la calidad de la gestión en instituciones universi- 
tarias, lleva consigo un cambio; se ha pasado de una visión burocrática del sistema a una visión más autónoma. Esta mayor autonomía implica una mayor y mejor evaluación de las instituciones y una perspectiva diferente que supone, además de una verificación del funcionamiento del sistema, un método para mejorarlo, con el fin de demostrar que tanto las decisiones tomadas, como las acciones ejecutadas están dirigidas a la mejora de la calidad. Todo esto unido a la exigencia de la calidad de los ciudadanos en todos los procesos productivos y en los servicios, la competitividad económica y la relativa estabilidad tanto financiera como de alumnado, parece indicar que ha llegado el momento de preocuparse por la mejora en la calidad de los procesos de gestión llevados a cabo en las instituciones universitarias.

\section{Antecedentes Teóricos}

La evaluación de la gestión directiva sólo adquiere sentido cuando se incorpora como práctica sistemática a un dominio más amplio que constituye la cultura institucional. La construcción de esta cultura permite la reflexión permanente sobre el papel de la universidad en la sociedad, la promoción sistemática de la innovación y la consolidación de nuevos modos de gestión y de relación con la comunidad. En este sentido, la evaluación pasa a ser un instrumento estratégico para la actualización constante de la universidad.

La institucionalización de la evaluación es, por su carácter de conquista cultural, una tarea larga. No puede ser el resultado de una decisión súbita, precedida de una reflexión insuficiente. Tampoco puede ser una construcción unilateral. Es un esfuerzo que debe asumir la totalidad de la comunidad universitaria.

Uno de los componentes principales de la calidad de la educación es, sin duda alguna, la evaluación concebida como un proceso complejo por medio del cual se pueden reorientar, mejorar o perfeccionar todas aquellas actividades que se relacionan con el aprendizaje, la enseñanza y el modo de operar los programas, estrategias y acciones del sector educativo.

En la búsqueda de calidad y de una identidad que le permita diferenciarse de otras instituciones, la universidad debe evaluar sus competencias y averiguar cuál es el método más adecuado para optimizar los procesos administrativos y de gestión que le permitirán generar una ventaja comparativa y competitiva en el contexto de las exigencias actuales del mercado. (Tejedor cit in Martínez, 2000).

\section{Competencias}

La producción intelectual sobre competencias laborales y los sistemas de certificación asociados, se han incrementado significativamente durante los últimos cinco años, sin que hasta la fecha exista un conjunto unívoco de definiciones universalmente aceptadas. La noción misma de competencia ejemplifica este estado de cosas, como lo testimonian las múltiples acepciones, alcances y matices dados al concepto. En palabras de Leboterf (1995) las competencias no son ellas mismas recursos en la forma de saber actuar, saber hacer o actitudes, más bien movilizan, integran y orquestan tales recursos. Esa movilización solo es pertinente en una situación, y cada situación es singular, mismo que pueda tratársela en analogía con otras, ya encontradas. El ejercicio de la competencia pasa por situaciones mentales complejas, esquemas de pensamiento que permiten determinar (más o menos consciente y rápidamente) y realizar (de modo más o menos eficaz) una acción relativamente adaptada a una situación. Se reconoce la insistencia en el papel movilizador de la competencia, es decir, una movilización desde el saber a la acción durante la cual se agrega valor en la forma de reacciones, decisiones y conductas exhibidas ante el desempeño. En este sentido un gestionador competente no se manifiesta en virtud de la capacidad de llevar a cabo instrucciones, sino más bien requiere además la actuación, es decir el valor añadi- 
do que el individuo competente pone en juego y que le permite saber encadenar unas instrucciones y no sólo aplicarlas aisladamente. En una concepción dinámica, las competencias se adquieren (educación, experiencia, vida cotidiana), se movilizan, y se desarrollan continuamente y no pueden explicarse y demostrase independientemente de un contexto. La competencia de un gestionador de calidad, está en la cabeza de éste, es parte de su acervo y su capital intelectual y humano.

En este sentido el enfoque basado en competencias puede ampliarse al plano colectivo y organizacional, logrando que las informaciones manejadas en la organización, por parte del directivo, y de los restantes miembros de la organización, se conviertan en conocimientos aprovechables para mejorar la competitividad. Las universidades, como toda organización, generan, almacenan y administran una gran cantidad de información, que deben convertir en conocimiento aplicable a la generación de innovaciones. La competencia individual, grupal y organizacional se convierte en un poderoso motor del aprendizaje y por ende en un aspecto fundamental en la gestión del recurso humano.

Las competencias consisten en cualquier característica individual o colectiva que se pueda medir de manera fiable, que se pueda demostrar y que diferencie de manera significativa a los trabajadores $\mathrm{u}$ organizaciones que mantienen un desempeño excelente (competencias diferenciadoras) de aquellos o aquellas que tienen un desempeño adecuado (competencias umbral), o entre los trabajadores o instituciones eficaces o ineficientes (Hooghiemstra, 1996, en Fernández Ríos, 1999).

Estos criterios de referencia son muy importantes para la definición de las competencias. Una de sus características es que no pueden considerarse como tales si no predicen algo significativo en el mundo real; "una diferencia que no diferencie no es una diferencia" (Spencer \& Spencer, 1993, en Alles 2000, p. 63), es decir, una característica que no provoque una diferencia en el rendimiento no es un criterio de referencia y no se puede utilizar para evaluar a las personas.

Los modelos más consolidados en Australia, Canadá y los Estados Unidos han propuesto siete competencias básicas, 3 de las cuales se pueden agrupar en diferentes habilidades.

En su totalidad las competencias básicas o genéricas, que se presentan a continuación a manera de ejemplo, apoyan la práctica profesional, e internacionalmente se han aplicado en la educación y en los ambientes laborales. Se ajustan a diferentes situaciones, circunstancias y ambientes (Argudín, 2001), a saber:

Competencias de estimación e injerencia, que se relacionan y dependen de los conocimientos de la disciplina; y dominio de tareas y contenidos. Competencias de comunicación que implican habilidades verbales, habilidades de lectura, habilidades de expresión escrita y habilidades de para manejar las nuevas tecnologías de información. Competencias de pensamiento crítico, que permiten realizar evaluación, análisis, resolución de problemas, toma de decisiones, y consulta. Competencias de relación, asociadas a actitudes relacionadas con el humanismo y el aspectos ético-valóricos, la cultura, relaciones interdisciplinares, trabajo en equipo y relaciones interpersonales. Competencias de función, referida a las habilidades para administrar (organizar, coordinar), planificar, uso de recursos, trato con el personal y responsabilidad. Competencia de liderazgo, que implica colaborar, planear y uso de la creatividad, por último, la Competencia de integrar conocimientos en desde y en relación con otras disciplinas y del entorno.

\section{Calidad en los Procesos de Gestión}

El término Gestión puede definirse como la capacidad de generar una relación adecuada entre la estructura, la estrategia, los sistemas, el estilo, las capacidades, la gente, y los objetivos superiores de la organización, o 
más simplemente como "La capacidad de articular los recursos de que se disponen de manera de lograr lo que se desea". (Casassus, 2000, p.286).

La gestión de la calidad ha sido siempre una de las máximas preocupaciones de las instituciones de educación superior, los interesados en la formación o mejor, los clientes de las instituciones, desean que la formación recibida corresponda con las habilidades y competencias demandadas en el trabajo. Ante la cada vez mayor demanda por formación y ante las rápidas y cambiantes condiciones, se ha hecho necesario que los oferentes de educación terciaria y perfeccionamiento demuestren ante la sociedad un trabajo bien hecho.

El análisis de la calidad en la formación se puede hacer desde el punto de vista de la gestión de la institución bajo el supuesto de que una organización que cumpla los principios implícitos en la norma, asegurará consistentemente la calidad de sus productos. (Vargas, 2003).

Implementar una gestión de calidad conlleva la aplicación de varios principios básicos: Una clara orientación al cliente, que permita comprender y satisfacer sus necesidades; una mejora continua de lo que hace la institución, con procesos definidos y consistentes que garanticen su cumplimiento; evaluación continua de la calidad de los productos y procesos y prevenir en lugar de supervisar, dado que los costos de medidas preventivas son menores que los de una estrecha supervisión.

La aplicación de los principios de calidad constituye una forma de dirección para hacer de las instituciones educativas entidades más eficientes, tanto para el desarrollo de sus funciones, como para la conformación de ambientes de trabajo competitivos que impliquen retos constantes para quienes participan en ellos y se reflejen en el logro de mejores niveles de desarrollo. (Alles, 2000).
Los principios de la Calidad Total, definida como: "un sistema efectivo para la integración de los esfuerzos de desarrollo, mantenimiento y mejoramiento que los diferentes grupos de una organización realizan para poder proporcionar un producto o servicio en los niveles más económicos para la satisfacción de las necesidades del usuario" (Feigenbaum cit in Bustos, 2002, p. 3), son en general difíciles de implantar en cualquier organización y, además presentan una particular complejidad cuando se trata de aplicarlos a los centros educativos, como son las universidades.

Al respecto, un modelo propuesto por Gento Palacios (1996), intenta presentar una visión objetiva de lo que deben ser las instituciones educativas de calidad. Considera a la calidad de estas instituciones en un enfoque integral totalizador, señalando a los componentes que conforman el centro o la institución educativa, como sujetos de análisis, a partir de criterios de calidad establecidos, formando parte de un sistema institucional, como una unidad que permitan resultados a partir de que se conjugan los elementos que la conforman. De tal forma que dichos componentes apoyen el efecto global de una manera integral, concurriendo sus elementos de una manera dinámica. Estos elementos son los indicadores de calidad y los predictores de calidad. Esta visión del modelo se fundamenta en consideraciones teóricas y empíricas, donde los diversos indicadores expresan la calidad, a la vez que permiten conocer el perfil de calidad de una institución, considerándoles como variables dependientes o criterios para conocer los resultados. El reflejo de la calidad en el perfil se da a partir de los predictores que permiten asegurarlo.

Se consideran indicadores de calidad, en las instituciones educativas, al producto educativo, lo que permite reconocer la calidad de la institución y sus componentes; la satisfacción de los estudiantes; la satisfacción del personal que labora en el centro educativo y 
el efecto que esto tiene en la educación. Un sistema de Gestión de Calidad para una universidad, según Kells, 1997, debería: Centrarse en la institución, es decir, su enfoque primario debe ser hacia el interior, ser de una naturaleza tal que favorezca el desarrollo, de tal manera que el mejoramiento y la ayuda sean su primera preocupación, ser íntegro (honesto) y responsablemente transparente, ser cíclico y cubrir los aspectos principales de la institución en cada ciclo, incluir la oportunidad de validación externa de los juicios internos, estar adecuadamente financiado, de manera que provea tanto incentivos marginales como fondos para los procesos de desarrollo de calidad y para las mejoras recomendadas, ser responsable ante diversos estamentos universitarios

La garantía de calidad por lo general implica la comparación entre un determinado producto o servicio y un estándar, previamente definido, que establece los criterios para calificar la calidad de dicho desempeño.

En este ámbito el estándar crecientemente utilizado en las organizaciones o empresas productivas o de servicios es la norma ISO. Esta norma se refiere a la garantía de calidad en una perspectiva general, no específicamente asociada a un producto o servicio determinado. Los consumidores son el punto de partida. Actualmente hay una alta valoración sobre las normas ISO como un "sello de calidad" y esta valoración ha hecho que se extienda su uso a las universidades.

Es en esta perspectiva que cualquier esfuerzo tendiente a evaluar y mejorar los procesos de gestión universitaria resulta relevante pensando en el futuro de estas instituciones de educación superior.

\section{Planteamiento del Problema}

La construcción de un instrumento capaz de evaluar la calidad de la gestión de directivos universitarios resulta relevante de ser analizado e investigado, dado que, pretende cubrir el vacío existente en la práctica administrativa al interior de las instituciones de educación superior.

La gran cantidad de alternativas académicas existentes actualmente en el mercado, pone de manifiesto la necesidad de contar con mecanismos de evaluación que enfaticen aquellos factores o dimensiones clave, que permitirían medir y eventualmente, guiar las acciones de los profesionales que ocupan cargos directivos al interior de las universidades.

Las consideraciones anteriores quedan de manifiesto en las siguientes hipótesis de investigación:

- Es posible construir y validar un instrumento para evaluar la calidad de la gestión de directivos universitarios.

- Se puede medir válidamente la calidad de la gestión de directivos universitarios mediante una Escala de Evaluación de la Gestión Directiva (E.E.G.D.).

El objetivo general, por ende, fue diseñar y validar la escala E.E.G.D., para evaluar la calidad de la Gestión de directivos de Instituciones Universitarias. Esto implicó analizar teóricamente el constructo Gestión Directiva Universitaria, elaborar un banco de ítemes en función de las competencias asociadas al constructo gestión Directiva y construir, con ellos, una versión preliminar del instrumento integrando los ítemes en dimensiones homogéneas, someter a validación de contenido el instrumento mediante juicio de expertos, analizar la confiabilidad de la escala y someter el instrumento definitivo a un proceso de validación de constructo mediante análisis factorial.

\section{Método}

\section{Tipo y diseño de investigación}

Para lograr los objetivos propuestos se postuló realizar una investigación de carácter descriptivo con un diseño de investigación 
transversal-correlacional, dado que se pretendía examinar la forma en que se percibe el fenómeno analizado "gestión directiva" en el marco de instituciones universitarias, y del mismo modo, cómo se correlacionan internamente sus componentes o dimensiones, en función de las opiniones expresadas por funcionarios universitarios ligados en primera línea de jerarquía respecto del cargo de Director de Departamento, de Carrera u otros.

\section{Población}

La población objetivo estuvo constituida por aquellos sujetos que trabajan en instituciones universitarias y que pertenecen a la línea directa en el organigrama, tanto ascendente como descendente respecto de la persona que ocupa el cargo denominado Director de Departamento, en términos genéricos. Por ende, desde el punto de vista ascendente la población está constituida por quien es el superior inmediato del Director, generalmente quien ocupa el cargo genérico de Decano de Facultad, y desde el punto de vista descendente, son todos los docentes, con jornada contratada, superior a media jornada, que pertenecen al Departamento evaluado.

\section{Muestra}

El procedimiento de muestreo fue no probabilístico casual o de sujetos voluntarios, dado que se debió contar con la voluntad de éstos para participar. En función de los requerimientos del análisis factorial proyectado, se consideró acceder a un número determinado de Decanos que evaluaron a 53 Directores de Departamento y un total de 100 docentes que evaluaron Directores.

A pesar de que existe una variedad de instrumentos que son utilizados en evaluación de gestión institucional a nivel de organizaciones, la mayor parte de ellos, carecen de un examen acucioso de su validez y confiabilidad. La generalidad, utiliza el criterio de expertos como un paso único para legitimar dicho proceso, dando cuenta escasamen- te de la validez de contenido de los reactivos, estímulos o preguntas que componen el instrumento utilizado.

La mayor parte de los estudios realizados en el área, son más bien de carácter descriptivo o exploratorio. Muchos de ellos han involucrado la participación de directivos de educación superior, sin embargo ésta se ha enfocado, principalmente, en la selección o detección de competencias relevantes para el ejercicio sus propias funciones.

Estudios como el de Stalcup y Wilson en 1981 o Townsend y Bassoppo-Moyo en 1997, son un ejemplo de ello. En dichas investigaciones, más que pretender evaluar cuantitativamente el nivel de desarrollo de competencias de gestión directiva en los sujetos seleccionados, se empleó la técnica de encuesta para aunar criterios respecto de las competencias más relevantes, a fin de implementarlas en programas curriculares destinados a la formación de directivos.

En nuestro país la realidad es más precaria, por lo que esta investigación se constituye en un aporte relevante, tanto en lo que respecta a la definición de competencias directivas universitarias, como en la rigurosidad del análisis metodológico a la base de los ítems propuestos para su evaluación.

\section{Procedimiento de Análisis de datos}

El diseño de este Instrumento de evaluación se realizó en tres etapas, comenzando con el proceso de Validez de Contenido, para posteriormente analizar, a través de su consistencia interna, el nivel de Confiabilidad de la Escala. Finalmente se procedió a evaluar la dimensionalidad de ésta, para verificar hasta qué punto los ítemes sugeridos permitían medir un constructo común.

\section{Validez de Contenido}

Una primera etapa de análisis fue depurar un conjunto de rasgos, atribuibles a quien posee competencias claves en el área de la 
Tabla 1. Dimensiones propuestas y distribución de reactivos por cada una de ellas.

\begin{tabular}{|cccc|}
\hline Dimensión & $\begin{array}{c}\text { Número de reactivos } \\
\text { o ítemes }\end{array}$ & Dimensión & $\begin{array}{c}\text { Número de reactivos } \\
\text { o ítemes }\end{array}$ \\
\hline Competencia Directiva & 12 & Trabajo en equipo & 12 \\
Liderazgo & 9 & Competencia cognitiva & 10 \\
Lenguaje y comunicación & 9 & Competencia valórica/actitudinal & 9 \\
\hline
\end{tabular}

Gestión y presentados en términos de conductas explicitas en su hacer. Estas conductas representan en qué grado dichos rasgos o indicadores cubren la variedad de significados incluidos en el concepto.

Este conjunto de rasgos, se obtuvo a partir del examen exhaustivo de la bibliografía relativa al tema, del cual se infirió un conjunto de competencias ligadas al constructo gestión directiva. Posteriormente, se procedió a explicitarlas en conductas observables ligadas al hacer específico de quien ocupa un cargo de tal naturaleza, ello permitió contar en principio con un total de 126 rasgos. En una segunda etapa, las competencias y los rasgos ligados a ellos, fueron subsumidos en dimensiones de carácter omniabarcantes, en esa oportunidad se determinó en principio un total de seis dimensiones, a saber:

Esta primera versión del instrumento, fue sometida a un proceso de validación de jueces expertos, que contó con la participación de 14 evaluadores, 7 mujeres y 7 varones, que compartían características tales como: haber ejercido, o estar desempeñándose en cargos directivos, con una experiencia mínima de 2 años, al interior de alguna Universidad del país. Este criterio les facultaba para emitir un juicio adecuado respecto de la pertinencia o no de los rasgos y dimensiones propuestas. Una vez recepcionado el material reportado por los jueces y procesado adecuadamente, su análisis permitió disminuir a cuatro las dimensiones originales, reorganizando y agrupando las dimensiones Competencia Direc- tiva, Lenguaje y Comunicación, lo mismo que las dimensiones Liderazgo y Trabajo en Equipo. Del mismo modo, se modificó en forma sustantiva la redacción de algunos reactivos o rasgos y se incorporó otros que pertenecían a la dimensión en función de la opinión experta de los jueces y que no habían sido considerados previamente. Ello permitió, finalmente construir la versión: Escala de Evaluación de la Gestión Directiva (EEGD), constituida por las dimensiones siguientes:

Gestión Institucional : 11 reactivos

Liderazgo y Trabajo

en Equipo : 10 reactivos

Cognitiva $\quad: 9$ reactivos

Valórica-Actitudinal : 12 reactivos

Las respuestas a los reactivos se presentaron en formato tipo Likert, e incluyeron 5 opciones:

1. Nada

2. Levemente

3. Moderadamente

4. Bastante

5. Absolutamente

Adicionalmente se incorporó la categoría "no corresponde", para identificar aquellos reactivos que no se evaluarán, por no representar a juicio de quien responde características atribuibles a la gestión del cargo. 


\section{Confiabilidad: Consistencia interna}

En la presente investigación se optó por analizar la consistencia interna de la escala, que describe estimaciones de confiabilidad basadas en la correlación promedio entre reactivos de una prueba y que resume la cantidad de error de medición esperada al usar el instrumento en una población dada, por tal motivo se utilizó el coeficiente alfa de Cronbach.

\section{Evaluación de la Dimensionalidad de la Escala: Análisis Factorial Exploratorio}

Una segunda etapa fundamental para el propósito de validación, era examinar si las dimensiones sugeridas y deducidas en el análisis previo, constituyen efectivamente los componentes del constructo Gestión Directiva Universitaria. Para ello y dado que, en este particular caso, no emerge con claridad un criterio con el cual establecer su validez predictiva, se optó por realizar un análisis factorial. Ahora bien, como cualquier procedimiento multivariado o psicométrico, es necesario combinar puntajes de diversas variables para obtener medidas válidas de constructo, por lo que se requiere algún método para determinar la legitimidad de combinaciones particulares. Los patrones de las correlaciones entre variables son muy importantes para determinar esta legitimidad. (Nunnally \& Bernstein 1995).

\section{Resultados}

Para tal efecto, se llevó a cabo un análisis factorial en dos etapas. Una primera fase exploratoria, utilizando un análisis de componentes principales, con rotación Varimax, con el propósito de identificar el número ini- cial de factores, que subyacen al constructo. En una segunda etapa se realizó un análisis factorial de eje principal, también con rotación Varimax, destinada a precisar los resultados anteriores. Se verificó que la matriz de correlaciones Inter-item, poseía un numero sustantivo de correlaciones significativas sobre $\mathrm{r}=0,3$, observándose la gran mayoría con coeficientes de correlación sobre $r=0,6$.

Posteriormente se utilizó el coeficiente MAS, medida de adecuación muestral, que permite verificar la pertinencia de los ítemes para formar parte del instrumento. Todos los valores observados cumplieron con el criterio $\geq 0,5$.

\section{Análisis de Componentes Principales: Varimax}

A través del análisis de Componentes Principales, se observó que el cálculo del determinante de la matriz de correlaciones, arrojó un valor $=3,62 * 10^{-26}$ el que resultó óptimo, pues es cercano a cero. Por ende las correlaciones observadas se deben a la asociación entre los ítemes. Seguidamente, se analizó el índice KMO (Medida de Adecuación Muestral de Kaiser, Meyer y Olkin), cuyo criterio de ser mayor que 0,5 se cumplió, dado que el valor alcanzado fue 0,951. Conjuntamente se aplicó el test de Esfericidad de Bartlett que resultó significativo, con un valor de 6139,838 . Cabe destacar que dicho valor resulta significativo al 99\%. Verificados los supuestos se admite la existencia de factores comunes.

En la tabla $\mathrm{N}^{\mathrm{o}} 2$ se resume el resultado de la distribución de valores propios y de la varianza total explicada.

Tabla 2. Distribución de los valores propios y varianza total explicada

\begin{tabular}{|cccc|}
\hline Valores Propios & Total & \% de Varianza & \% Acumulativo \\
\hline 1 & 24,037 & 57,231 & 57,231 \\
\hline 2 & 1,903 & 4,531 & 61,763 \\
\hline 3 & 1,307 & 3,113 & 64,875 \\
\hline 4 & 1,185 & 2,821 & 67,697 \\
\hline
\end{tabular}


Se observa que existen a los más cuatro factores principales dados que sus valores son superiores a 1, según regla de Kaiser. Conjuntamente, el análisis de gráfico de sedimentación, confirma que existen a lo más dos o tres factores, y a lo menos uno principal. Lo anterior, debido a que éste último test, da cuenta de que existe un número limitado de factores comunes sustantivos y un número de factores comunes triviales, medidas por las variables del instrumento.

Con estos antecedentes, se revela la misma estructura original del test, y por ende se procedió a analizar soluciones para 4, 3 y 2 factores respectivamente. No obstante, en la presente investigación sólo se informa los resultados más importantes de analizar, correspondientes a la solución de 2 y 4 factores. Con la finalidad de encontrar la solución más pertinente, se replicó el análisis de componentes principales con rotación Varimax. En la tabla $\mathrm{N}^{\mathrm{o}} 3$, se presenta la matriz de cargas para la solución de cuatro y dos factores respectivamente, en la cual se han eliminado los valores inferiores $\mathrm{a}=0,45$. 
Tabla 3. Matriz de cargas factoriales para cuatro y dos factores

\begin{tabular}{|c|c|c|c|c|c|c|}
\hline $\begin{array}{c}\text { Dimensiones y Número } \\
\text { de Item }\end{array}$ & \multicolumn{4}{|c|}{ Soluciones de 4 Factores } & \multicolumn{2}{|c|}{ Solución de 2 Factores } \\
\hline & 1 & 2 & 3 & 4 & 1 & 2 \\
\hline \multicolumn{7}{|l|}{ G.I. } \\
\hline 1 & .615 & & .475 & & .579 & .466 \\
\hline 2 & .666 & & .497 & & .642 & .489 \\
\hline 3 & .623 & & & & .649 & \\
\hline 4 & .576 & & & & .684 & \\
\hline 5 & .466 & & .564 & & .497 & .589 \\
\hline 6 & .641 & .459 & & & .753 & \\
\hline 7 & .637 & & & & .754 & \\
\hline 8 & & .466 & & & .529 & \\
\hline 9 & .603 & & & .472 & .653 & \\
\hline 10 & .549 & & & & .685 & \\
\hline 11 & .467 & & & & .510 & \\
\hline \multicolumn{7}{|l|}{ L. y T.E. } \\
\hline 12 & .671 & & & & .653 & \\
\hline 13 & .633 & & & & .705 & \\
\hline 14 & .772 & & & & .742 & \\
\hline 15 & & .741 & & & & .586 \\
\hline 16 & & .597 & & & .557 & .473 \\
\hline 17 & & .635 & & & .591 & .528 \\
\hline 18 & & .597 & & & .577 & \\
\hline 19 & & .686 & & & .639 & \\
\hline 20 & .623 & & & & .707 & \\
\hline 21 & & & .464 & & .525 & .611 \\
\hline \multicolumn{7}{|l|}{ C. } \\
\hline 22 & .620 & .460 & & & .653 & \\
\hline 23 & .622 & .518 & & & .711 & \\
\hline 24 & .468 & .589 & & & .592 & .471 \\
\hline 25 & .505 & .480 & & & .627 & .564 \\
\hline 26 & .627 & & & & .651 & .477 \\
\hline 27 & .773 & & & & .759 & \\
\hline 28 & .605 & & & & .700 & \\
\hline 29 & .496 & & .492 & & .547 & .555 \\
\hline 30 & .578 & & & & .637 & \\
\hline \multicolumn{7}{|l|}{ V.A. } \\
\hline 31 & & & .821 & & & .779 \\
\hline 32 & & & .815 & & & .757 \\
\hline 33 & & & .558 & & & .693 \\
\hline 34 & & .568 & .546 & & & .708 \\
\hline 35 & & & & & .570 & .556 \\
\hline 36 & & & & & .572 & .539 \\
\hline 37 & .549 & & & & .662 & .491 \\
\hline 38 & & & .749 & & & .754 \\
\hline 39 & & & & .459 & .481 & .554 \\
\hline 40 & & & .609 & & & .766 \\
\hline 41 & & & & .734 & & \\
\hline 42 & & & & .688 & & .594 \\
\hline
\end{tabular}


Tabla 4. Distribución de los valores propios y varianza total explicada

\begin{tabular}{|c|c|c|c|}
\hline Valores Propios & Total & \% de Varianza & \% Acumulativo \\
\hline 1 & 24,037 & 57,231 & 57,231 \\
\hline 2 & 1,903 & 4,531 & 61,763 \\
\hline
\end{tabular}

Los valores de cargas consignados en la tabla $\mathrm{N}^{\mathrm{o}} 3$ y la de los valores propios y varianza total explicada de la tabla $\mathrm{N}^{\mathrm{o}} 4$ permiten señalar que efectivamente, existen dos Factores, un componente principal y un factor secundario que subyacen en su estructura. A continuación, se procedió a tratar de reducir los ítemes del primer factor, no obstante, que todos ellos cumplieron con el criterio de carga mínima superior a $=0,45$. Se optó por seleccionar sólo aquellos que poseían una carga superior a $=0,60$, y examinar como se comportaba dicha solución, con la reducción en el número de ítemes, verificando si se ajustaba estadísticamente. Estos factores fueron: factor I (itemes 2, 3, 4, 6, 7, 9, $10,12,13,1419,20,22,23,24,25,26,27,28$,
37), factor II (itemes 31, 32, 33, 34, 35, 36, 38, $39,40)$.

Para aquellos ítemes que cargaron favorablemente a los dos factores, se utilizó un criterio teórico de pertinencia para su inclusión en uno de los dos factores, en paralelo a su mayor carga.

El Indice de Adecuación Muestral KMO, fue de 0,949 y el Test de Esfericidad de Bartlett arrojó un 4118,285.

El valor de la Determinante $=2,291 \mathrm{E}-13$

En la tabla $\mathrm{N}^{\circ} 5$ se muestra los valores propios y su distribución por factor, lo mismo que la varianza total explicada.

Tabla 5. Distribución de los valores propios y varianza total explicada

\begin{tabular}{|c|c|c|c|c|c|c|}
\hline Factor & Total & $\begin{array}{c}\text { \% de } \\
\text { Varianza }\end{array}$ & $\begin{array}{c}\text { \% } \\
\text { Acumulativo }\end{array}$ & Total & $\begin{array}{c}\text { \% de } \\
\text { Varianza }\end{array}$ & $\begin{array}{c}\text { \% } \\
\text { Acumulativo }\end{array}$ \\
\hline 1 & 17,208 & 59,338 & 59,338 & 16,842 & 58,075 & 58,075 \\
\hline 2 & 1,791 & 6,175 & 65,513 & 1,439 & 4,961 & 63,036 \\
\hline 3 & 1,000 & 3,447 & 68,960 & & & \\
\hline 4 &, 860 & 2,964 & 71,924 & & & \\
\hline
\end{tabular}

Tabla 6. Distribución de los valores propios y varianza total explicada para dos factores.

\begin{tabular}{|c|c|c|c|}
\hline Valores Propios & Total & \% de Varianza & Acumulativo \% \\
\hline 1 & 11,201 & 38,626 & 38,626 \\
\hline 2 & 7,079 & 24,410 & 63,036 \\
\hline
\end{tabular}


Tabla 7. Matriz de Cargas Factoriales para dos factores

\begin{tabular}{|c|c|c|}
\hline & Factor 1 & Factor 2 \\
\hline P27 & ,766 & \\
\hline P7 & ,762 & \\
\hline P6 & ,756 & \\
\hline P14 & ,745 & \\
\hline $\mathrm{P} 23$ & 730 & \\
\hline P13 & 717 & \\
\hline P20 & ,716 & \\
\hline P28 & 698 & \\
\hline P4 & 693 & \\
\hline P37 & 684 & ,466 \\
\hline P10 & 681 & \\
\hline P22 & ,667 & \\
\hline P26 & ,665 & ,461 \\
\hline P3 & 659 & \\
\hline P12 & 656 & \\
\hline P2 & ,653 & ,483 \\
\hline P19 & 651 & \\
\hline P25 & ,649 &, 534 \\
\hline P9 & 648 & \\
\hline P24 & 607 & \\
\hline P35 & ,591 & ,534 \\
\hline P36 & ,585 &, 528 \\
\hline P31 & & 807 \\
\hline P32 & & 776 \\
\hline P38 & & 762 \\
\hline $\mathrm{P} 40$ & & ,733 \\
\hline P34 & & 681 \\
\hline P33 & & 675 \\
\hline P39 & ,505 & ,507 \\
\hline
\end{tabular}

El análisis anterior, (tablas 5, 6 y 7) con el subconjunto de itemes seleccionado, confirma un factor principal al que denominare- mos Gestión Institucional Global, y un factor secundario que denominaremos Valórico Actitudinal. 
Análisis de la Confiabilidad y

Discriminación de itemes

Se examinó la consistencia interna del instrumento y su homogeneidad, mediante el coeficiente Alfa de Cronbach, la correlación ítem-total corregido (ver tabla 9) mostró los siguientes valores, por cada factor/dimensión y la confiabilidad de la escala en su conjunto.

Tabla 8. Indices de confiabilidad del instrumento por Factor y en forma global

\begin{tabular}{|l|c|}
\hline \multicolumn{1}{|c|}{ Dimensión } & Indice Alfa de Cronbach \\
\hline Gestión Institucional Global & aa $=0,9694$ \\
\hline Valórica-Actitudinal & aa $=0,9333$ \\
\hline Total & aa $=0,9751$ \\
\hline
\end{tabular}

Como puede observarse de las tablas anteriores, los índices de confiabilidad del instrumento son altamente satisfactorios, (Nunnally \& Bernstein 1995), tanto a nivel de factores o dimensiones, como a nivel global. Ello permite inferir que las variabilidades observadas en los puntajes de los individuos, respecto del constructo gestión de directivos universitarios se debe a diferencias individuales y no a factores ligados al azar.

La tabla siguiente expone los índices de discriminación de los ítemes a considerar por cada factor:

Tabla 9. Indices de Discriminación de los ítemes por factor

\begin{tabular}{|c|c|c|c|}
\hline \multicolumn{2}{|c|}{ Factor I } & \multicolumn{2}{c|}{ Factor II } \\
\hline Itemes & Indice de Discriminación & Itemes & Indice de Discriminación \\
\hline P2 &, 8017 & P31 &, 7533 \\
\hline P3 &, 7603 & P32 &, 7022 \\
\hline P4 &, 7855 & P33 &, 7899 \\
\hline P6 &, 7554 & P34 &, 7673 \\
\hline P7 &, 7903 & P35 &, 7582 \\
\hline P9 &, 7333 & P36 &, 7405 \\
\hline P10 &, 7117 & P38 &, 7525 \\
\hline P12 &, 7176 & P39 &, 6888 \\
\hline P13 &, 8122 & P40 &, 8269 \\
\hline P14 &, 7079 & & \\
\hline P19 &, 7541 & & \\
\hline P20 &, 8053 & & \\
\hline P22 &, 7972 & & \\
\hline P23 &, 8206 & & \\
\hline P25 &, 8136 & & \\
\hline P26 &, 7955 & & \\
\hline P27 &, 8138 & & \\
\hline P28 &, 7437 & & \\
\hline P24 &, 7357 & & \\
\hline P37 &, 7983 & & \\
\hline
\end{tabular}

$N^{o}$ de Casos $=153,0 \quad N^{o}$ de Items $=20$ y 9 
Se observa que los índices de discriminación son todos satisfactorios y discriminan adecuadamente, (Nunnally \& Bernstein 1995) Es decir, quienes emiten percepciones favorables en el reactivo, tienden a alcanzar los más altos puntajes en la escala en su conjunto.

\section{Discusión y Proyecciones}

Diseñar, construir y validar un instrumento de medición en el ámbito de las ciencias humanas es de por sí un proceso complejo, no sólo por la secuencia rigurosa de pasos que la constituyen, sino adicionalmente, por la naturaleza epistemológica inherente a tales ciencias, pues los constructos que se intentan objetivar no poseen las características de los llamados objetos físicos y, aún más, los instrumentos que se utilizan para examinar su presencia, no gozan de un consenso unánime, ni poseen la condición de norma única contra la cual contrastar una eventual observación particular. Más bien, las condiciones de construcción y validación, comportan la adherencia a ciertos supuestos necesarios para lograr la rigurosidad requerida por un instrumento que se ponga a disposición de la comunidad científica en el área. En este orden de cosas, el estudio tuvo como objetivo central configurar un instrumento que examinara el constructo teórico "Gestión de Directivos Universitarios", ello implicó en primer lugar realizar una exhaustiva revisión teórica que permitiera acercarse a tal fenómeno, con el propósito de derivar de este sustrato teórico referencial elementos constitutivos sólidamente respaldados de lo que se denomina comúnmente Gestión. Se optó por referir los principales enfoques sobre el particular, y adherir al enfoque o modelo basado en competencias, como el eje orientador del proceso de construcción de rasgos o conductas visibles o exteriorizables, congruentes con las competencias constitutivas o fundamentales del constructo.

Se postuló que quien desempeña un cargo de naturaleza directiva, en la cual debe elucidar competencias de gestión, puede ser evaluado en su desempeño por quienes forman parte de su flujo jerárquico directo tanto ascendente como descendente, en una perspectiva de evaluación de $360^{\circ}$ grados. Ello permitió identificar la población objetivo para aplicar el instrumento, funcionarios universitarios jefes directos de directores de Departamento o afines, generalmente Decanos, y funcionarios docentes, bajo el mando directo del Director de Departamento, que reciben el desempeño de su gestión y que por ende, están en condiciones de emitir un juicio sobre tal función. Al respecto, cabe mencionar que al tratarse de una muestra voluntaria, es decir, no probabilistica, se debe ser cautelosos a la hora de generalizar los hallazgos a una población con características diferentes a la estudiada. Lo mismo ocurre al considerar el tamaño muestral y su relación con los reactivos iniciales, proporción que no se ajusta rigurosamente a la mínima sugerida en la literatura para realizar un análisis factorial. Esto también nos conduce a ser cautelosos en la interpretación de los resultados.

Por la importancia y relevancia del análisis factorial realizado, éste se detalla en profundidad. El cual se llevó a cabo en dos etapas. Una primera fase exploratoria con el propósito de identificar el número de factores que subyacen al constructo. En la segunda etapa se realizó un análisis factorial de eje principal.

Se verificó el cumplimiento de los supuestos fundamentales para la aplicación de dicho análisis, a saber: que la matriz de correlaciones Inter-item demostró que éstas son altamente significativas; que el coeficiente de adecuación muestral (MAS), era verificado para cada uno de los itemes del instrumento, y que, por ende, demuestran su pertinencia; el valor del determinante de la matriz es cercano a cero $(3,632$ E-20); el índice KMO alcanzó un valor $=0,951$, superior al criterio de 0,5 y también se constató que el Test de Esfericidad de Bartlet, es significativo. $(p<0,01)$. 
Verificados estos supuestos, conjuntamente se observó la existencia inicial de cuatro factores en función de la regla de Kaiser y el gráfico de Sedimentación. Con todos estos antecedentes se analizó en forma secuenciada, la pertinencia de soluciones para tres y dos factores subyacentes en el instrumento. Las matrices de cargas factoriales sucesivas, permitieron decidir por la opción definitiva de dos Factores. Uno principal denominado Gestión Institucional Global, y otro secundario, denominado Dimensión ValóricoActitudinal.

La serie de análisis intermedios, permitieron entre otras cosas eliminar en forma definitiva el item 41, de la versión sometida a análisis, (genera instancias para celebrar actividades de reconocimiento y/o fechas significativas para la unidad o miembros de ella), pues no cargaba lo suficiente hacia ningún factor, además, teóricamente aparece justificada su exclusión. El resto de los ítemes, si poseían carga significativa, algunos de ellos se recomponen y refusionan en un factor inclusivo, denominado Gestión Institucional Global, así las dimensiones iniciales propuestas Liderazgo y Trabajo en Equipo y Dimensión Cognitiva, se subsumieron en el gran factor que emergió del análisis. Este factor, quedó conformado por 20 reactivos, en función de poseer una carga superior $\mathrm{a}=0,6 \mathrm{de}$ un conjunto de 33 con cargas superiores $\mathrm{a}=$ 0,45 . Los ítemes definitivos de esta nueva dimensión son: $2,3,4,6,7,9,10,12,13,14$, $19,20,22,23,24,25,26,27,28,37$. En el caso de este último item (transmite entusiasmo y confianza estimulando el desafío por descubrir y emprender nuevos proyectos o posibilidades), se incorpora a la dimensión Gestión institucional Global, dado que su lectura posibilita que el estímulo está referido más a la creación o la oportunidad de contar con un espacio para generar actividad propia o proyectos particulares, que una cuestión de incentivo valórico. La decisión de disminuir la cantidad de ítemes, está también avalada y justificada, por el tipo de población usua- rio u objetivo del instrumento, constituidos por profesionales de instituciones de educación superior, que generalmente no poseen una disponibilidad de tiempo que le permita responder con rigurosidad y disposición instrumentos con un número importante de ítemes, como eran los 42 iniciales, además, la nueva versión permite un enfrentamiento a los reactivos o estímulos-rasgos bastante homogéneo, pues se concentran en dos grandes dimensiones. Ello repercute favorablemente en la practicidad del instrumento, transformándose en una escala operativa desde el punto de vista de su abordaje, predisponiendo a responderla, pues no se percibe como tediosa y extensa.

El segundo Factor, referido a aspectos Actitudinales y Valóricos, quedó configurado por 9 reactivos, a saber: 31, 32, 33, 34, 35, $36,38,39,40$. todos ellos con cargas superiores $\mathrm{a}=0,45$, en el caso de los ítemes 35, 36, 39 , ellos también cargan al factor Gestión Institucional Global, pero se mantienen en la categoría por una decisión teórica, respaldada también por la carga estadística probada.

Analizada esta última solución de factores con los ítemes descritos y seleccionados, fue sometida de nuevo al análisis factorial, observándose que la solución se ajusta en forma pertinente.

Los índices de confiabilidad y de discriminación de los ítemes son altamente significativos. Lo que respalda su estructura final, la que puede observarse detalladamente en su versión última que se adjunta al final de este apartado.

A estas características fundamentales inherentes a todo instrumento de medición social que se precie de tal, se suma el enorme potencial de contar con una escala de evaluación que posee la condición de ser aplicada en forma ascendente y descendente, respecto del Directivo evaluado en cuanto a su gestión, ello posibilita recoger un cúmulo de información de capital importancia para 
aquellas instituciones que permanentemente examinan la calidad de sus procesos formativos y organizacionales. Contar con este tipo de bi-direccionalidad, permite recoger la percepción de los subordinados inmediatos, la más de las veces, absolutamente ausentes de emitir una opinión válidamente considerada. Poder ponderar justipreciadamente dichas percepciones y acometer la tarea de examinar sus congruencias y evidencias reportadas parece ser un elemento de gran aporte para iniciar procesos de mantención y/o optimización. Además, de constituir un aporte verdaderamente original en el ámbito de la gestión, donde no existe un instrumento que examine el constructo de acuerdo a las características de la presente escala, basada en un enfoque de competencias.

En la actualidad las universidades nacionales e internacionales, están llevando a cabo evaluaciones de la calidad de sus procesos, entre los cuales se cuentan: exámenes acuciosos de sus mallas curriculares, procesos de autoevaluación y evaluación de pares externos, del mismo modo, comienza a institucionalizarse la necesidad de acreditar las carreras, programas de formación inicial o de post-grado; como también examinar rigurosamente la calidad de los profesionales que egresan de sus aulas. Ello ha implicado examinar el tipo de información entregada y los agentes que la reportan, con la finalidad de generar un proceso que sea a lo menos consensuado, por las diversas autoridades y potenciales clientes de dichas instituciones. En este proceso variadas han sido las técnicas para recopilar información pertinente, desde la objetivización de variables tipo, tales como: metros cuadrados versus estudiantes, número de textos por alumno, jornadas de docentes contratadas, puntajes de admisión, media de egreso, índices de deserción, AFI, publicaciones, grados, recursos económicos, etc. Con esta finalidad, se recurre a mecanismos diversos, desde estadísticas y publicaciones periódicas, hasta entrevistas, aplicaciones de escalas, Focus, técnicas cualitativas, etc.
En este orden de cosas, el aporte que constituye esta investigación fue proveer un instrumento que examinara la gestión directiva y su evidente relación con la calidad de los procesos internos y externos de una institución de educación superior. Esta investigación puede iniciar o dar pie a un estudio exhaustivo de variables específicas ligadas a un mejor desempeño institucional, y por qué no señalarlo, el liderazgo que podrían ellas comenzar a generar. Además, el enfoque basado en competencias, permite examinar y evaluar la relación de rasgos evidenciables en conductas específicas que pueden presentar niveles de desempeño diversos. Ello podría redundar, en reconocer fortalezas y debilidades institucionales, con una perspectiva de mejoramiento intrínseco más que inicialmente coercitivo. Especialmente, cuando las actuales políticas de desarrollo universitario, postulan la búsqueda permanente de recursos externos, de carácter concursable u otros, que propendan a la racionalización económica, debido a que los aportes estatales resultan cada vez más insuficientes. Un buen gestionador debería evidenciar competencias claves en esta área.

En la medida que la organización tenga conocimiento de las competencias presentes en los directivos universitarios, será posible evaluar su desempeño, lo que dará paso a un certero perfeccionamiento y plan de incentivo, que motive las acciones que optimicen los procesos de gestión y el cumplimiento de estándares de calidad previamente establecidos por la organización. De esta manera, el óptimo desempeño de quienes dirigen estas instituciones, posibilitará un clima apropiado que fomente la motivación de las personas, satisfacción laboral y el consecuente compromiso organizacional, facilitando la consecución de la calidad total como un objetivo claro y compartido.

En este sentido, se puede proyectar la realización de investigaciones que examinen por ejemplo, la siguiente hipótesis sugerente: 
"Una institución con un clima organizacional óptimo o adecuado, se relaciona directamente con puntajes altos de sus autoridades directivas en el ámbito de la gestión y muy especialmente en la dimensión Valórica Actitudinal".

Del mismo modo, examinar si el lugar ocupado en Rankings de universidades publicados últimamente se correlaciona con puntajes altos de sus autoridades directivas en la escala.

Otra línea de desarrollo investigativo la constituye, el poder estandarizar los puntajes obtenidos en la escala, fijando criterios de desempeño jerarquizables, en función de una muestra relevante de evaluaciones de Gestión de Directivos universitarios. Señalando intervalos de puntajes que discriminen intensidades de desempeño ascendente.

También se plantea la posibilidad de correlacionar los resultados del test como variable específica de Gestión, con otras variables delámbito de la Psicología organizacional o del ámbito de la productividad, o el grado de satisfacción de alumnos o funcionarios.

\section{Referencias}

Aguilera, S.( 2002). La cultura de la evaluación. Recuperado el 17 de Agosto de 2003 en http://www.observatorio.org/colaboraciones/aguilera.html

Alles, M. (2000). Dirección estratégica de recursos humanos, gestión por competencias. Buenos Aires: Gránica.

Argudín, Y. (2001). Educación basada en Competencias. Recuperado el 17 Agosto de 2003 en http://www. Jalisco. Gob.Mx/srias/educacion / consulta / educar / 19/ argudin. Html

Bustos, A et al. (2002). Diseño de un instrumento para evaluar competencias claves en el desempeño de Directivos de Instituciones
Universitarias. Tesis para optar al grado de Licenciado en Psicología: U. San Sebastián, Concepción.

Casassus, J. (2000). Problemas de la gestión educativa en América Latina. Revista Pensamiento Educativo. 2, 282-286.

Fernández-Ríos, M. (1999). Diccionario de recursos humanos. Organización y dirección. Madrid: Díaz de Santos.

Kells, H. (1997). Procesos de Autoevaluación. Una guía para la Autoevaluación en la Educación Superior. Lima: Fondo Editorial de la Pontificia Universidad Católica del Perú.

LeBoterf (1995). De la Compétence. Essai sur un atracteur ètrange. [Ensayo sobre un extracto extraño]. Paris: Les éditios d'Organisation.

Martínez, R. (2000). Evaluación de la Gestión Universitaria. Barcelona: Síntesis.

Martínez, R. (2000). Evaluación de la Gestión Universitaria. Informe preparado para la CONEAU. Recuperado el 18 Agosto de 2003 en http://www.coneau.edu.ar/ que_es / document/publicaciones / Evaluacion_gestion_universitaria. PDF

Nunnally, J. \& Bernstein, I. (1995). Teoría psicométrica. México: Mc. Graw hill.

Palacios, G. (1996). Instituciones educativas para la calidad total. Madrid: La Muralla.

Pérez, M. \& Requena, A. (2000). Evaluación Institucional de la Facultad de Ciencias Políticas y Sociología de la Universidad de Granada. Revista de Educación, Cuestiones Escolares Ministerio de Educación Cultura y Deporte. 18, 420-437.

Rebolloso, E. Et. Al. (2001). Estrategias de calidad en la Universidad. Valencia: Promolibro.

Townsend, B \& Bassoppo-Moyo(1997). The effective community college academic 


\section{Escala de Evaluación de la Gestión Directiva (EEGD)}

\section{Dimensión Gestión Institucional Global}

1. Efectúa planificaciones basadas en una sólida comprensión de la misión global de la Universidad, sus políticas y prácticas.

2. Coordina esfuerzos de planificación con otros estamentos universitarios.

3. Identifica posibles problemas de la institución y la repercusión de éstos en los componentes de la unidad.

4. Fomenta la modificación de los planes de acción o las estrategias cuando éstas no son funcionales a la organización.

5. Se mantiene alerta a los cambios del entorno, detectando necesidades de reestructuración, innovación o mejoramiento que incorpore valor a la unidad.

6. Clarifica roles y funciones de los miembros de la unidad u organización según corresponda.

7. Comunica en forma clara y precisa, a los miembros de su unidad, cuestiones relativas a la marcha institucional, la toma de decisiones o tareas específicas, cuando corresponde o se le solicita.

8. Establece y comunica estándares de calidad que permiten retroalimentar efectivamente el desempeño de los miembros de la unidad.

9. Dirige al grupo hacia objetivos y metas definidas otorgando tiempo y recursos necesarios para su logro.

10. Diseña mecanismos de control, seguimiento del rendimiento y productividad del personal a su cargo evaluando las acciones de mejora en torno a los resultados obtenidos.

11. Demuestra capacidad para persuadir o influenciar al personal a su cargo respecto de los objetivos que ha definido.

12. Visualiza las potencialidades y capacidades de su personal para el logro de los objetivos de la organización

13. Analiza objetivamente los problemas, considerando sus implicaciones y alternativas antes de tomar decisiones.

14. Visualiza, evalúa y decide las alternativas de solución más convenientes ante los problemas que enfrenta.

15. Ante presiones laborales puede pensar con claridad, mantener sus productividad, sin perder el foco de la problemática.

16. Demuestra creatividad y flexibilidad en la toma de decisiones y en el abordaje de las problemáticas.

17. Analiza reflexivamente el desarrollo de su trabajo.

18. Anticipa los problemas y cambios en el entorno, planificando y desarrollando respuestas con proactividad e innovación.

19. Posee capacidad de evaluar la información que recibe de los diferentes niveles y actores de la organización, y decidir cursos de acción al respecto.

20. Trasmite entusiasmo y confianza, estimulando el desafío por descubrir y emprender nuevos proyectos o posibilidades.

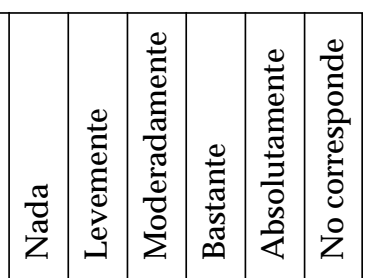

\begin{tabular}{l|l|l|l|l}
1 & 2 & 3 & 4 & 5
\end{tabular}

5

$\begin{array}{lllllll}1 & 2 & 3 & 4 & 5\end{array}$

$\begin{array}{lllllll}1 & 2 & 3 & 4 & 5\end{array}$

\begin{tabular}{l|l|l|l|l|}
1 & 2 & 3 & 4 & 5
\end{tabular}

\begin{tabular}{l|l|l|l|l}
1 & 2 & 3 & 4 & 5
\end{tabular}

\begin{tabular}{l|l|l|l|l|l}
1 & 2 & 3 & 4 & 5
\end{tabular}

$\begin{array}{lllllll}1 & 2 & 3 & 4 & 5\end{array}$

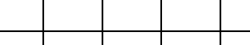

\begin{tabular}{l|l|l|l|l}
1 & 2 & 3 & 4 & 5
\end{tabular}

$\begin{array}{lllllll}1 & 2 & 3 & 4 & 5\end{array}$

$\begin{array}{llllll}1 & 2 & 3 & 4 & 5\end{array}$

$\begin{array}{lllllll}1 & 2 & 3 & 4 & 5\end{array}$

\begin{tabular}{l|l|l|l|l|l}
1 & 2 & 3 & 4 & 5
\end{tabular}

\begin{tabular}{l|l|l|l|l|l|}
1 & 2 & 3 & 4 & 5
\end{tabular}

\begin{tabular}{l|l|l|l|l|l}
1 & 2 & 3 & 4 & 5
\end{tabular}

\begin{tabular}{l|l|l|l|l|l}
1 & 2 & 3 & 4 & 5
\end{tabular}

\begin{tabular}{lll|l|l|}
\hline & & & \\
\hline
\end{tabular}

$\begin{array}{lllllll}1 & 2 & 3 & 4 & 5\end{array}$

\begin{tabular}{l|l|l|l|l|l}
1 & 2 & 3 & 4 & 5
\end{tabular}

\begin{tabular}{ll|l|l|l|l}
1 & 2 & 3 & 4 & 5
\end{tabular}

\begin{tabular}{l|l|l|l|l}
1 & 2 & 3 & 4 & 5
\end{tabular}

\begin{tabular}{l|l|l|l|l}
1 & 2 & 3 & 4 & 5
\end{tabular}




\begin{tabular}{|c|c|c|c|c|c|c|}
\hline Dimensión Valórica - Actitudinal & $\begin{array}{l}\frac{\pi}{0} \\
\frac{\pi}{Z}\end{array}$ & 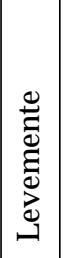 & 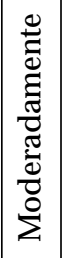 & 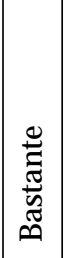 & 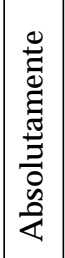 & 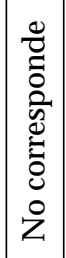 \\
\hline $\begin{array}{l}\text { 21. Es capaz de mantener un equilibrio entre los valores morales y éticos de la } \\
\text { organización y su visión personal acerca de ellos. }\end{array}$ & 1 & 2 & 3 & 4 & 5 & \\
\hline 22. Actúa conforme a las normas y a los estándares éticos de la organización. & 1 & 2 & 3 & 4 & 5 & \\
\hline $\begin{array}{l}\text { 23. Demuestra interés y comprende los problemas que pueden estar } \\
\text { afectando a sus colaboradores y/ o pares. }\end{array}$ & 1 & 2 & 3 & 4 & 5 & \\
\hline $\begin{array}{l}\text { 24. Muestra actitudes constructivas y asertivas en sus relaciones } \\
\text { interpersonales al interior de la universidad. }\end{array}$ & 1 & 2 & 3 & 4 & 5 & \\
\hline $\begin{array}{l}\text { 25. Es capaz de involucrar a los demás en las decisiones que puedan afectar al } \\
\text { clima y cultura Organizacional. }\end{array}$ & 1 & 2 & 3 & 4 & 5 & \\
\hline $\begin{array}{l}\text { 26. Apoya y promueve el desarrollo personal de los miembros de la unidad a } \\
\text { su cargo. }\end{array}$ & 1 & 2 & 3 & 4 & 5 & \\
\hline 27. Es capaz de respetar a quienes no comparten sus valores e ideas. & 1 & 2 & 3 & 4 & 5 & \\
\hline $\begin{array}{l}\text { 28. Estimula a los miembros de su organización a que planteen sus } \\
\text { desacuerdos y/o criticas de forma directa y constructiva. }\end{array}$ & 1 & 2 & 3 & 4 & 5 & \\
\hline $\begin{array}{l}\text { 29. Procura mantener un clima de trabajo sano, ofreciendo un espacio para } \\
\text { escuchar las inquietudes, necesidades y sugerencias de los colaboradores. }\end{array}$ & 1 & 2 & 3 & 4 & 5 & \\
\hline
\end{tabular}

Fecha Recepción Artículo: 15 de Septiembre de 2004

Fecha Evaluación Final: $\quad 21$ de Diciembre de 2004 